\title{
The anomalous anomaly
}

\author{
Understanding the muon's magnetic moment holds the key for unlocking potential new physics, as \\ Thomas Teubner shows.
}

n classical electrodynamics, the magnetic moment of a particle can be understood as arising from a spinning charge distribution. However, even point-like particles have a magnetic moment $\boldsymbol{\mu}$ that is aligned with their spin $\mathbf{s}: \boldsymbol{\mu}=g(q / 2 m) \mathbf{s}$ with $q$ and $m$ the charge and mass of the particle, respectively, and $g$ its so-called g-factor.

From his theory of relativistic quantum mechanics, Paul Dirac predicted that the g-factor of elementary spin-1/2 particles, such as the electron, is exactly 2 . At first, this seemed to be in agreement with atomic physics experiments, but in 1947 small deviations from the expectations were seen in the hyperfine structure of hydrogen and deuterium. A small deviation of the electron's g-factor from 2 was proposed as an explanation. In 1948 Julian Schwinger calculated that in quantum electrodynamics (QED), fluctuations lead to a tiny change of $g$. His famous result, the so-called anomalous magnetic moment, $a=(g-2) / 2=\alpha /(2 \pi)$, where $\alpha \approx 1 / 137$ is the fine structure constant, is engraved on his tombstone and solved the puzzle.

Schwinger's result was just the starting point of a big success story. The further development of QED is very closely connected with ever more accurate predictions for $g-2$ using perturbation theory. Today, the state of the art is the inclusion of corrections up to five so-called loops (virtual exchanges with photons and fermions - Schwinger did one loop where just one photon is exchanged). In 2012, after many years' work, Toichiro Kinoshita and collaborators finished the calculation of 12,672 Feynman diagrams $^{1}$; their five-loop contribution is proportional to $(\alpha / \pi)^{5}$, which is very small but still relevant when comparing experiment and theory. The anomalous magnetic moment (also simply called 'anomaly') of the electron, $a_{\mathrm{e}}$, is measured to an astonishing 0.25 parts per billion and provides a very accurate determination of $\alpha$.

So why were physicists turning to the muon, the more than 200 times heavier sibling of the electron? First, a precise measurement of its anomaly tests if the

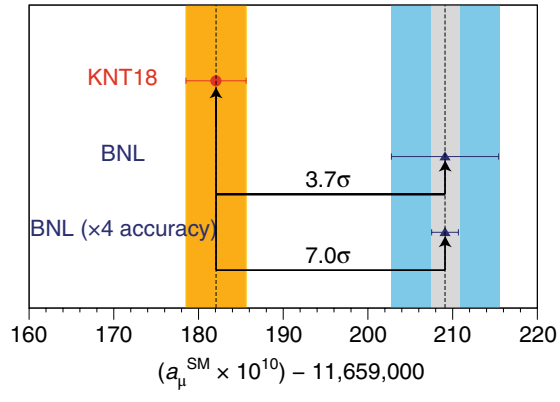

muon is a fundamental particle or has a composite structure. Second, the muon's anomaly, $a_{\mu}$, is not only an effect from QED, but also contains significant contributions from the weak and strong forces of the standard model (SM) of particle physics. Moreover, any unknown particle or force in nature could contribute in addition to the known ones. For yet undiscovered heavy particles, the muon anomaly would pick up such effects much more strongly compared to the electron, typically by a factor of the muon mass over the electron mass, squared $(\approx 43,000)$. Therefore, studying the muon with high precision tests our quantum understanding of nature, including the yet unknown.

Early measurements of $a_{\mu}$ at CERN confirmed theoretical predictions, and some influential scientists predicted that nothing interesting would ever be learned from this. Others disagreed and pushed for more accurate measurements. At the turn of the millennium, experiment E821 at Brookhaven National Laboratory (BNL) measured $a_{\mu}$ with an accuracy of 0.5 parts per million, much better than before ${ }^{2}$.

Their result disagreed with the theoretical predictions, with a statistical significance of two to three standard deviations. Over the past two decades, this discrepancy, despite various small changes and twists, has never gone away, and has led to a lot of excitement and hundreds of theoretical ideas seeking an explanation in terms of 'new physics'.

In recent years, all aspects of the SM theory prediction for $a_{\mu}$ have been scrutinized and refined. With continued theoretical and computational efforts, its uncertainty has now become smaller than the experimental error of the BNL measurement. In turn, the discrepancy has been further consolidated and now stands at $3.7 \sigma\left(\mathrm{KNT} 18\right.$; ref. $\left.{ }^{3}\right)$. The figure shows the current discrepancy of $3.7 \sigma$ with a possible future increase to $7 \sigma$, due to a projected four-times improved experimental accuracy if the mean value stays unchanged.

To decide beyond doubt whether the discrepancy is real, new, more precise measurements of $a_{\mu}$ are needed. The nextgeneration experiment E989 at Fermilab is under way (http://muon-g-2.fnal.gov). It is designed to measure $a_{\mu}$ with about four times improved accuracy. Also, a second, completely different and independent experiment is currently being built at J-PARC in Japan, planned to start operation in a few years' time (http://g-2.kek.jp).

E989 has already recorded first data that the collaboration is now feverishly analysing, while at the same time preparing the experiment to collect data with much better statistics. The results are eagerly awaited; if a discrepancy is fully confirmed at a higher statistical significance, this would be a clear sign for physics beyond the standard model (BSM). Conversely, if after all these efforts there would be agreement between measurements and SM theory, then this would exclude many BSM models. Either way, taming the muon's anomaly is key in driving our physics understanding forward.

\section{Thomas Teubner \\ Department of Mathematical Sciences, University of Liverpool, Liverpool, UK. \\ e-mail: thomas.teubner@liverpool.ac.uk}

Published online: 1 November 2018

https://doi.org/10.1038/s41567-018-0341-3

References

1. Aoyama, T., Hayakawa, M., Kinoshita, T. \& Nio, M. Phys. Rev. Lett. 109, 111808 (2012).

2. Bennett, G. W. et al.(Muon g-2 Collaboration) Phys. Rev. D 73, 072003 (2006).

3. Keshavarzi, A., Nomura, D. \& Teubner, T. Phys. Rev. D 97 114025 (2018).

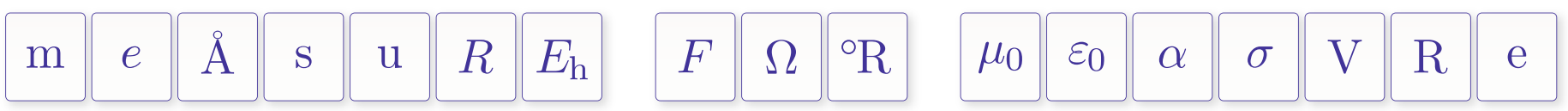

